University of Texas at El Paso

ScholarWorks@UTEP

$7-2020$

\title{
It Is Important to Take All Available Information into Account When Making a Decision: Case of the Two Envelopes Problem
}

\author{
Laxman Bokati \\ The University of Texas at El Paso, Ibokati@utep.edu \\ Olga Kosheleva \\ The University of Texas at El Paso, olgak@utep.edu \\ Vladik Kreinovich \\ The University of Texas at El Paso, vladik@utep.edu
}

Follow this and additional works at: https://scholarworks.utep.edu/cs_techrep

Part of the Applied Mathematics Commons

Comments:

Technical Report: UTEP-CS-20-85

\section{Recommended Citation}

Bokati, Laxman; Kosheleva, Olga; and Kreinovich, Vladik, "It Is Important to Take All Available Information into Account When Making a Decision: Case of the Two Envelopes Problem" (2020). Departmental Technical Reports (CS). 1479.

https://scholarworks.utep.edu/cs_techrep/1479

This Article is brought to you for free and open access by the Computer Science at ScholarWorks@UTEP. It has been accepted for inclusion in Departmental Technical Reports (CS) by an authorized administrator of ScholarWorks@UTEP. For more information, please contact Iweber@utep.edu. 


\title{
It Is Important to Take All Available Information into Account When Making a Decision: Case of the Two Envelopes Problem
}

\author{
Laxman Bokati, Olga Kosheleva, and Vladik Kreinovich
}

\begin{abstract}
In situations when we know the probabilities of all possible consequences, traditional decision theory recommends selecting the action that maximizes expected utility. In many practical situations, however, we only have partial information about the corresponding probabilities. In this case, for different possible probability distributions, we get different values of expected utility. In general, possible values of expected utility form an interval. One way to approach this situation is to use the optimism-pessimism approach proposed by Nobelist Leo Hurwicz. Another approach is to select one of the possible probability distributions - e.g., the one that has the largest possible entropy. Both approaches have led to many good practical applications. Usually, we get reasonable conclusions even when we ignore some of the available information - e.g., because this information is too vague to be easily formalized. In this paper, we show, on the example of the two envelopes problem, that ignoring available information can lead to counter-intuitive recommendations.
\end{abstract}

\section{Decision Making Under Imprecise Probabilities: A Brief Reminder}

Decision theory: a brief reminder. Traditional decision theory (see, e.g., $[1,6,8$, $10,12]$. ) is based on the notion of utility. The natural-language word "utility" has many meanings, but in decision theory, it has a very precise - and somewhat unusual - meaning.

To describe this meaning, we need to select two alternatives:

- a very bad alternative $A_{-}$which is worse that anything that we will actually encounter, and

Laxman Bokati, Olga Kosheleva, and Vladik Kreinovich

University of Texas at El Paso, El Paso, Texas 79968, USA

e-mail: lbokati@miners.utep.edu, olgak@utep.edu,vladik@utep.edu 
- a very good alternative $A_{+}$which is better than anything that anything that we will actually encounter.

Then, for each number $p$ from the interval $[0,1]$, we can form a lottery $L(p)$ in which:

- we get $A_{+}$with probability $p$ and

- we get $A_{-}$with the remaining probability $1-p$.

For $p=0$, the lottery $L(p)$ coincides with the very bad alternative $A_{-}$and is, thus, worse that any actual alternative $A$; we will denote this by $L(0)=A_{-}<A$. For $p=1$, the lottery $L(p)$ coincides with the very good alternative $A_{+}$and is, thus, better than any actual alternative $A: A<A_{+}$.

We can ask the user to compare the alternative $A$ with the lotteries $L(p)$ corresponding to different probabilities $p$. We assume that for every two alternatives $A$ and $B$, the user always decides:

- whether $A$ is better (i.e., $B<A$ )

- or whether $B$ is better (i.e., $A<B$ ),

- or whether $A$ and $B$ are of the same quality to this user; we will denote this by

$$
A \sim B .
$$

We also assume that the user's decisions are consistent - i.e.:

- that the preference relation $<$ is transitive and

- that $p<p^{\prime}$ implies $L(p)<L\left(p^{\prime}\right)$.

One can see that under these assumptions, there is a threshold value $p_{0}$ such that:

- for all $p<p_{0}$, we have $L(p)<A$, and

- for all $p<p_{0}$, we have $A<L(p)$.

This threshold value is called the utility of the alternative $A$. The utility is usually denoted by $u(A)$.

By definition of the utility, for every small value $\varepsilon>0$, we have

$$
L(u(A)-\varepsilon)<A<L(u(A)+\varepsilon) .
$$

For very small $\varepsilon$, lotteries with probabilities $u(A)$ and $u(A) \pm \varepsilon$ are practically indistinguishable, so we can interpret the above double inequality as saying that the alternative $A$ is equivalent to the lottery $L(u(A))$. We will denote this equivalence by

$$
A \equiv L(u(A)) .
$$

Suppose now that for some action $a$, we have consequences $A_{1}, \ldots, A_{n}$ with probabilities $p_{1}, \ldots, p_{n}$. This means that the action $a$ is equivalent to a lottery in which we get each $A_{i}$ with probability $p_{i}$. Each alternative $A_{i}$, in its turn, is equivalent to the lottery $L\left(u\left(A_{i}\right)\right)$ in which we get $A_{+}$with probability $u\left(A_{i}\right)$ and $A_{-}$with the remaining probability $1-u\left(A_{i}\right)$. Thus, the action $a$ is equivalent to a two-stage lottery, in which: 
- first, we select $A_{i}$ with probability $p_{i}$, and

- then, depending on which alternative we selected on the first stage, we select $A_{+}$ with probability $u\left(A_{i}\right)$ and $A_{-}$with probability $1-u\left(A_{i}\right)$.

As a result of this two-stage lottery, we get either $A_{+}$or $A_{-}$, and the probability of getting $A_{+}$is equal to $p=\sum_{i=1}^{n} p_{i} \cdot u\left(A_{i}\right)$. So, the action $a$ is equivalent to the lottery $L(p)$. By definition of utility, this means that the utility $u(a)$ of the action $a$ is equal to this probability $p$, i.e., that

$$
u(a)=\sum_{i=1}^{n} p_{i} \cdot u\left(A_{i}\right)
$$

By definition of utility, we select the action with the largest possible value of utility. The right-hand side of the above formula is the expected value of the utility, so this means that a rational person should select the alternative with the largest possible value of expected utility.

Utility is defined modulo a linear transformation. Numerical values of utility depend on the selection of the very bad and very good alternatives $A_{-}$and $A_{+}$. What if we select a different pair $A_{-}^{\prime}$ and $A_{+}^{\prime}$ ?

For example, if $A_{-}<A_{-}^{\prime}<A_{+}^{\prime}<A_{+}$, then for every alternative $A$, its utility $u^{\prime}(A)$ in relation to $A_{-}^{\prime}$ and $A_{+}^{\prime}$ means that $A$ is equivalent to a lottery $L^{\prime}\left(u^{\prime}(A)\right)$ in which we get $A_{+}^{\prime}$ with probability $u^{\prime}\left(A_{+}\right)$and $A_{-}^{\prime}$ with the remaining probability $1-u^{\prime}(A)$. Here:

- the alternative $A_{-}^{\prime}$ is equivalent to a lottery $u\left(A_{-}^{\prime}\right)$ in which we get $A_{+}$with probability $u\left(A_{-}^{\prime}\right)$ and $A_{-}$with the remaining probability $1-u\left(A_{-}^{\prime}\right)$;

- similarly, $A_{+}^{\prime}$ is equivalent to a lottery $u\left(A_{+}^{\prime}\right)$ in which we get $A_{+}$with probability $u\left(A_{+}^{\prime}\right)$ and $A_{-}$with the remaining probability $1-u\left(A_{+}^{\prime}\right)$.

Thus, the original alternative $A$ is equivalent to a two-stage lottery in which:

- we first select $A_{-}^{\prime}$ or $A_{+}^{\prime}$, and

- then, depending on what we selected on the first stage, select $A_{+}$or $A_{-}$.

As a result of this two-stage lottery, we get either $A_{+}$or $A_{-}$, and the probability $p$ of selecting $A_{+}$is equal to

$$
p=u^{\prime}(A) \cdot u\left(A_{+}^{\prime}\right)+\left(1-u^{\prime}(A)\right) \cdot u\left(A_{-}^{\prime}\right)=u\left(A_{-}^{\prime}\right)+u^{\prime}(A) \cdot\left(u\left(A_{+}\right)-u\left(A_{-}\right)\right) .
$$

By definition of utility, this probability $p$ is the utility $u(A)$ of the alternative $A$ in terms of $A_{-}$and $A_{+}$. Thus,

$$
u(A)=u\left(A_{-}^{\prime}\right)+u^{\prime}(A) \cdot\left(u\left(A_{+}\right)-u\left(A_{-}\right)\right) .
$$

In other words, the utility is defined modulo a linear transformation.

Comment. This is similar to measuring quantities like time or temperature, where the numerical value depends on the selection of the starting point and of the measuring unit. 
What if we only have partial information about probabilities: two approaches. The formula for the expected utility assumes that we know the probability of each alternative. In many practical situations, we only have partial information about the probabilities. In this case, for different probability distributions $P=\left(p_{1}, \ldots, p_{n}\right)$, we have different values of the expected utility. If two probability distributions $P$ and $P^{\prime}$ are possible, then we can also consider as possible the case when we have $P$ with probability $\beta$ and $P^{\prime}$ with probability $1-\beta$. In this case, the expected utility is equal to the convex combination of expected utilities corresponding to $P$ and $P^{\prime}$. So, the set of possible values of expected utility is closed under convex combinations and is, thus, an interval

$$
[\underline{u}(a), \bar{u}(a)] .
$$

How can we make a decision under this interval uncertainty? There are two approaches to solving this problem.

The first approach is based on the fact that we need to compare the action $a$, in particular, with lotteries $L(p)$. Thus, we need to assign, to this interval, a corresponding utility $u(a)$ depending on the bounds $u(a)=f(\underline{u}(a), \bar{u}(a))$ for some functions $f(x, y)$. Utility is defined modulo a linear transformation $u(a) \rightarrow k \cdot u(a)+\ell$, for some $k>0$ and $\ell$. It is therefore reasonable to require that the function $f(x, y)$ not depend on the selection of the alternative $A_{-}$and $A_{+}$, i.e., that it remains the same for all $k>0$ and $\ell$ :

- if $z=f(x, y)$,

- then $z^{\prime}=f\left(x^{\prime}, y^{\prime}\right)$ for $z^{\prime}=k \cdot a+\ell, x^{\prime}=k \cdot x+\ell$, and $y^{\prime}=k \cdot y+\ell$.

In particular, every interval $[\underline{a}, \bar{a}]$ can be obtained from the interval $[0,1]$ if we take $k=\bar{u}-\underline{u}$ and $\ell=\underline{u}$. Thus, if we denote $\alpha \stackrel{\text { def }}{=} f(0,1)$, then the above invariance implies that

$$
f(\underline{u}, \bar{u})=\alpha \cdot(\bar{u}-\underline{u})+\underline{u}=\alpha \cdot \bar{u}+(1-\alpha) \cdot \underline{u} .
$$

So, when we make a decision, we should select the action $a$ for which the value

$$
\alpha \cdot \bar{u}+(1-\alpha) \cdot \underline{u}
$$

attains the largest possible value.

This idea was first proposed by a Nobelist Leo Hurwicz. It is known as Hurwicz optimism-pessimism approach; see, e.g., [2, 6, 8]. The reason for this name is as follows:

- When $\alpha=1$, this means that the decision maker only takes into account the best possible situation, with utility $\bar{u}$; this is what we usually mean by extreme optimism.

- Vice versa, when $\alpha=0$, this means that the decision maker only takes into account the worst possible situation, with utility $\underline{u}$; this is what we usually mean by extreme pessimism.

Of course, in real life, these two behaviors do not make sense: 
- extreme pessimism would mean not going into the street at all since there is a change of getting hit by a car, and

- extreme optimism would mean crossing the street on red light in heavy traffic.

Realistic decision making corresponds to values $\alpha$ between 0 and 1. For example, it is often recommended to select $\alpha=0.5$.

An alternative approach is that instead of considering all possible probability distributions, we should select the most reasonable one. Some of the possible distributions correspond to larger uncertainty, some to smaller uncertainty. We do not want to pretend that we have less uncertainty, so it is reasonable to select the distribution with the largest possible value of uncertainty. A natural measure of uncertainty of a probability distribution is its entropy - the average number of binary questions that we need to ask to determine the actual value; see, e.g., [3, 11]. Thus, out of all possible distributions, we select the one with the largest possible entropy. This approach is known as the Maximum Entropy approach; see, e.g., [3].

In particular, when we have a natural symmetry - e.g., we have no reason to believe that some alternatives are more probable than others - the resulting distribution is also invariant with respect to the same symmetry. So, if we class of distributions was invariant with respect to permutations, the maximum entropy distribution is also invariant with respect to all permutations - and thus, assigns equal probability to all alternatives. This is known as Laplace Indeterminacy Principle.

This invariance idea can be applied to the case when all we know is that the value $x$ of some physical quantity is positive, but we have no information about different probabilities. Which probability density function (pdf) $\rho(x)$ should we choose? The numerical value of $x$ depends on the choice of a measuring unit: if we select a unit which is $\lambda$ times smaller, then all numerical values $x$ are replaced by new values $x^{\prime}=\lambda \cdot x$. For example, 1.7 meters becomes $100 \cdot 1.7=170 \mathrm{~cm}$. Under this transformation, the original probability density function $\rho(x)$ takes, in the new unit, the form

$$
\frac{1}{\lambda} \cdot \rho\left(\frac{x^{\prime}}{\lambda}\right)
$$

We want to have the pdf that does not depend on the choice of the measuring unit, so we should have

$$
\frac{1}{\lambda} \cdot \rho\left(\frac{x^{\prime}}{\lambda}\right)=\rho\left(x^{\prime}\right)
$$

or, equivalently,

$$
\rho\left(\frac{x^{\prime}}{\lambda}\right)=\lambda \cdot \rho\left(x^{\prime}\right)
$$

For $x^{\prime}=1$ and $\lambda=1 / a$, we get $\rho(a)=$ const $\cdot \frac{1}{a}$.

It should be noted that, strictly speaking, this is not a probability density function, since here, we have $\int \rho(a) d a=\infty \neq 1$. This is known as an improper probability distribution. So, if we use a pr

What we do in this paper. Both Hurwicz and Maximum Entropy approaches have been used in many practical problems. They usually lead to intuitively acceptable 
results, even when we ignore some available information - e.g., when this information is too vague to be easily formalized.

In this paper, we show, on the example of the two envelopes problems, that if we ignore this information, both approaches lead to a counter-intuitive results. Thus, when making a decision under uncertainty, it is important to take into account all available information.

\section{Two Envelopes Problem: Description and Analysis}

Formulation of the problem. The two envelopes problem is as follows; see, e.g., [9] and references therein. Someone places some amount of money in one envelope, and a double that amount in another envelope. We do not know which envelope contains a smaller amount and which contains a larger amount.

We can pick one envelope and check how much money $x$ it has. Then, we can make a decision:

- we can either keep this amount

- or we can select the second envelope instead.

The question is:

- should we keep the original amount or

- should we select the other envelope instead?

How realistic is this situation. The above situation is over-simplified, but similar situations occur in real life.

For example, suppose that there are two competing countries producing certain military equipment. A smaller country would like to buy from one of them. For political reasons, it cannot negotiate simultaneously with both, so it starts serious negotiations with one of the countries. After negotiations, it comes up with the expected cost $x$ of the purchase. This cost is usually rather significant. Now, this smaller country has a choice:

- it can go with this contract, or

- it can try its luck by negotiating with the competing country - taking into account that if these negotiations do not lead to cheaper prices, there will be no going back to the original negotiated contract: e.g., the first weapons-producing country will have already committed its factories to produce weapons for someone else.

What should it do?

More peaceful examples can be found: e.g., when forming political alliances inside a country or within countries, when planning mergers, etc.

At first glance, this is somewhat paradoxical. Let us go back to the original two envelopes problem. From the common sense viewpoint, since our selection was random, it does not make sense to switch. 
On the other hand, intuitively, since we do not know these the second envelope has $2 x$ or $x / 2$. Thus, in line with the Laplace Indeterminacy Principle, we assume that these two amounts have equal probability 0.5 . But then, the expected gain is

$$
0.5 \cdot 2 x+0.5 \cdot(x / 2)=1.25 x
$$

Since $1.25 x>x$, it looks like it is always reasonable to switch - which contradicts to common sense.

Comment. This argument is based on the assumption that utility is proportional to money. In practice, it is proportional to square root of money (see, e.g., [4, 5, 7]), but the paradox remains if we take $\sqrt{2} \cdot x$ and $x / \sqrt{2}$ as the utilities of the two alternatives.

This is not really a paradox. A detailed analysis shows that, in reality, there is no paradox. Indeed, suppose that the original values come with the probability density $\rho(x)$. Then, the double amounts come with probability density $\frac{1}{2} \cdot \rho\left(\frac{x}{2}\right)$. Then, if we find the value $x$ :

- the condition probability that this is the original amount of money - and thus, that the remaining envelope will contain $2 x$-is proportional to $\rho(x)$, and

- the conditional probability that this is the double amount of money - and thus, that the remaining envelope contains $x / 2-$ is proportional to

$$
\frac{1}{2} \cdot \rho\left(\frac{x}{2}\right)
$$

Since these two probabilities should add up to 1 , we conclude that they should be equal to

$$
\frac{\rho(x)}{\rho(x)+\frac{1}{2} \cdot \rho\left(\frac{x}{2}\right)}
$$

and to

$$
\frac{\frac{1}{2} \cdot \rho\left(\frac{x}{2}\right)}{\rho(x)+\frac{1}{2} \cdot \rho\left(\frac{x}{2}\right)} .
$$

Thus, the expected value of the gain that we get when we switch is equal to

$$
\frac{\rho(x)}{\rho(x)+\frac{1}{2} \cdot \rho\left(\frac{x}{2}\right)} \cdot 2 x+\frac{\frac{1}{2} \cdot \rho\left(\frac{x}{2}\right)}{\rho(x)+\frac{1}{2} \cdot \rho\left(\frac{x}{2}\right)} \cdot \frac{x}{2} .
$$

So:

- If this amount is smaller that $x$, we should not switch.

- If this amount is larger than $x$, we should switch. 
- If this amount is equal to $x$, it does not matter whether we switch or not.

If we know the probability distribution, then, depending of the amount $x$, we should switch or not switch. For example:

- If we know that all the values $x$ are smaller than some amount $x_{0}$, then, if we get an amount $x>x_{0}$, we know that this is the larger amount, so switching does not make sense.

- Similarly, if we know that all original amounts are larger than or equal to some minimal amount $m$, and we have a value $x<2 m$, we know that this cannot be the double amount, so we should switch.

The above formula explains why there is no paradox: the only time when the probability of each option is exactly $1 / 2$ is when $\rho(x)=\frac{1}{2} \cdot \rho\left(\frac{x}{2}\right)$ for all $x$, and since this can be repeated not just for doubling money, but also for $\lambda$ times larger amount, we should have $\rho(x)=\frac{1}{\lambda} \cdot \rho\left(\frac{x}{\lambda}\right)$ for all $x$ and $\lambda$ - and we already know that this leads to an "improper" - not real - probability distribution.

Idealized formulation: what the two approaches recommend. Suppose now that we do not have any information about the probability distributions. What should the above two approaches recommend?

Let us first consider the Hurwicz approach. We do not know the pdf, so we should consider all possible probability density functions.

- The worst-possible case is when $\frac{1}{2} \cdot \rho\left(\frac{x}{2}\right)=0$, then after switching, we get $x / 2$ with probability 1 .

- The best-possible case is when $\rho(x)=0$, then after switching, we get $2 x$ with probability 1 .

So, e.g., for $\alpha=1 / 2$, the Hurwicz combination is equal to

$$
0.5 \cdot 2 x+0.5 \cdot(x / 2)=1.25 x>x
$$

so we arrive at the counter-intuitive conclusion that we should always switch.

So maybe we should use smaller value of Hurwicz's $\alpha$ ? This will not help, since, as we have mentioned, we could have $\lambda \cdot x$ in the second envelope. In this case, the expected value after switching is $\alpha \cdot \lambda \cdot x+(1-\alpha) \cdot(x / \lambda)$. For any $\alpha>0$, for a sufficiently large $\lambda$, we get a counter-intuitive conclusion that we should switch. The only case when this conclusion is not possible is the case $\alpha=0$ which, as we have mentioned, is not realistic at all. So, for this problem, Hurwicz approach leads to a counter-intuitive behavior.

Let us now consider the maximum entropy approach. Since all we know is that the amount is positive, we should use the corresponding improper distribution for which $\rho(x)=\frac{1}{2} \cdot \rho\left(\frac{x}{2}\right)$, so we also get $1.25 x$. In other words, in this case, the Maximum Entropy approach also leads to a counter-intuitive conclusion. 
So what should we do? Why did we get the counter-intuitive result? Because our description of the problem is not realistic. Do we really believe that an envelope can contain any amount of money? Realistically, an envelope cannot contain more than several thousand dollars - a million will not fit into an envelope. If we impose an upper limit $x_{0}$ on the original amount of money, then the conclusions change - and become more intuitive:

- For the Hurwicz approach with $\alpha=0.5$, we still recommend switching when $x \leq x_{0}$, but now we do not recommend switching when $x>x_{0}$, since in this case, $\rho(x)>0$.

- The Maximum Entropy approach leads to the uniform distribution

$$
\rho(x)=\text { const on the interval }\left[0, x_{0}\right] \text { and } \rho(x)=0 \text { for } x>x_{0} .
$$

For this distribution, the above formula also recommends switching when $x \leq x_{0}$ and not switching when $x>x_{0}$.

This common recommendation seems to be in perfect accordance with common sense.

Conclusion. When making decision under uncertainty, it is important to take into account all available information, even seemingly useless one - otherwise, if we ignore this information, we may end up with counter-intuitive recommendations.

\section{Acknowledgments}

This work was supported in part by the National Science Foundation grants 1623190 (A Model of Change for Preparing a New Generation for Professional Practice in Computer Science) and HRD-1242122 (Cyber-ShARE Center of Excellence).

\section{References}

1. P. C. Fishburn, Utility Theory for Decision Making, John Wiley \& Sons Inc., New York, 1969.

2. L. Hurwicz, Optimality Criteria for Decision Making Under Ignorance, Cowles Commission Discussion Paper, Statistics, No. 370, 1951.

3. E. T. Jaynes and G. L. Bretthorst, Probability Theory: The Logic of Science, Cambridge University Press, Cambridge, UK, 2003.

4. D. Kahneman, Thinking, Fast and Slow, Farrar, Straus, and Giroux, 2011.

5. O. Kosheleva, M. Afravi, and V. Kreinovich, "Why utility non-linearly depends on money: a commonsense explanation", Proceedings of the 4th International Conference on Mathematical and Computer Modeling, Omsk, Russia, November 11, 2016, pp. 13-18.

6. V. Kreinovich, "Decision making under interval uncertainty (and beyond)", In: P. Guo and W. Pedrycz (eds.), Human-Centric Decision-Making Models for Social Sciences, Springer Verlag, 2014, pp. 163-193. 
7. J. Lorkowski and V. Kreinovich, "Granularity helps explain seemingly irrational features of human decision making”, In: W. Pedrycz and S.-M. Chen (eds.), Granular Computing and Decision-Making: Interactive and Iterative Approaches, Springer Verlag, Cham, Switzerland, 2015, pp. 1-31.

8. R. D. Luce and R. Raiffa, Games and Decisions: Introduction and Critical Survey, Dover, New York, 1989

9. M. D. McDonnell and D. Abott, "Randomized switching in the two-envelope problem", Proceedings of the Royal Society A, 2009, Vol. 465, No. 2111, pp. 3309-3322.

10. H. T. Nguyen, O. Kosheleva, and V. Kreinovich, "Decision making beyond Arrow's 'impossibility theorem', with the analysis of effects of collusion and mutual attraction", International Journal of Intelligent Systems, 2009, Vol. 24, No. 1, pp. 27-47.

11. H. T. Nguyen, V. Kreinovich, B. Wu, and G. Xiang, Computing Statistics under Interval and Fuzzy Uncertainty, Springer Verlag, Berlin, Heidelberg, 2012.

12. H. Raiffa, Decision Analysis, McGraw-Hill, Columbus, Ohio, 1997. 